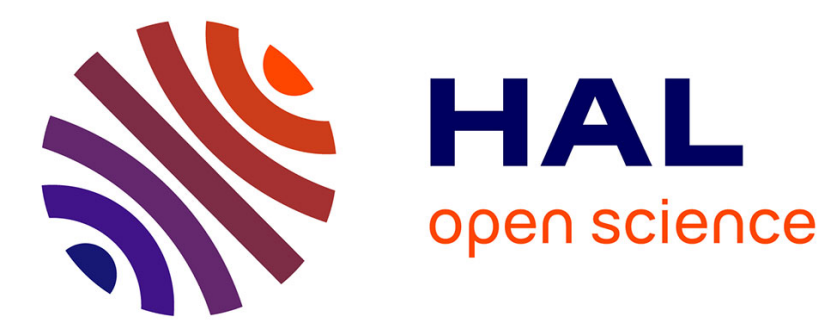

\title{
Les capteurs électrochimiques microniques
}

P. Clechet, N. Jaffrezic-Renault, C. Martelet

\section{To cite this version:}

P. Clechet, N. Jaffrezic-Renault, C. Martelet. Les capteurs électrochimiques microniques. Journal de Physique IV Proceedings, 1994, 04 (C1), pp.C1-283-C1-294. 10.1051/jp4:1994120 . jpa-00252462

\section{HAL Id: jpa-00252462 https://hal.science/jpa-00252462}

Submitted on 1 Jan 1994

HAL is a multi-disciplinary open access archive for the deposit and dissemination of scientific research documents, whether they are published or not. The documents may come from teaching and research institutions in France or abroad, or from public or private research centers.
L'archive ouverte pluridisciplinaire HAL, est destinée au dépôt et à la diffusion de documents scientifiques de niveau recherche, publiés ou non, émanant des établissements d'enseignement et de recherche français ou étrangers, des laboratoires publics ou privés. 


\title{
Les capteurs électrochimiques microniques
}

\author{
P. CLECHET, N. JAFFREZIC-RENAULT et C. MARTELET
}

Laboratoire de Physicochimie des Interfaces, Ecole Centrale de Lyon, BP. 163, 69131 Ecully cedex, France

\begin{abstract}
Presently, two main factors influence the evolution of both chemical and electrochemical sensors. The first is the very strong demand in sensors from various fields of industry. The second is the intrusion of microelectronic technologies in their fabrication processes which lead to the new concept of miniaturized sensors. The advantages of such small sensors are discussed. The various technologies of miniaturization used in the fabrication of electrochemical sensors are reviewed as well as the various methods of sensitization employed to functionalize the microtransducers, especially the Field Effect Transistors (FET).
\end{abstract}

\section{I - INTRODUCTION}

Les capteurs chimiques et biochimiques d'essence électrochimique (1) connaissent actuellement, comme du reste l'ensemble des capteurs, une véritable révolution conceptuelle aussi bien que technologique. Celle-ci résulte, essentiellement, de deux facteurs :

- le premier est la formidable demande dont ils font l'objet qui provient à la fois

- de la sévérité croissante des normes dans le domaine de l'environnement, de l'alimentation, de la pharmacie, de la sécurité industrielle, de la domotique...

- des besoins fabuleux en microcapteurs bio-compatibles ou non pour le monitoring et le diagnostic médical.

- de la généralisation des procédés d'automatisation dans le génie des procédés industriels.

- le deuxième est l'intrusion récente, mais en force, des technologies de miniaturisation diffusées par la microélectronique.

Ce dernier point, qui conditionne de plus en plus les moyens de fabrication des capteurs, est tout à fait décisif car il introduit des idées nouvelles quant à la constitution et aux méthodes d'utilisation de ceux-ci.

A la notion de macrocapteurs, c'est-à-dire de capteurs de taille centimétrique, souvent associés à une électronique assez lourde (l'électrode en verre en est un excellent exemple) et utilisés de façon unique, ce qui leur impose de présenter des performances individuelles remarquables : sensibilité, reversibilité mais surtout sélectivité, se substitue celle de microcapteurs, de taille au moins millimétrique, fabriqués par des procédés de masses et utilisés souvent de façon collective. De cette fabrication en masse sont attendues toute une série de qualités particulières allant de la réduction en taille et une fiabilité accrue (car c'est une conséquence classique des productions en grande série) à un coût très bas et surtout 
une aptitude naturelle au couplage avec les circuits intégrés de traitement du signal. Cette dernière possibilité ouvre alors la voie à deux conséquences considérables :

- d'une part à une facilité accrue de corriger le signal analytique des artéfacts pouvant provenir d'effets perturbants comme l'influence de la température ou autres stimulis. On obtient alors des capteurs capables de réagir spontanément, c'est-à-dire "intelligents".

- d'autre part à la possibilité de multiplexer plusieurs capteurs de façon à créer un réseau dont chaque élément répond de façon imparfaite aux différents constituants d'un mélange mais dont on peut extraire, par le calcul, le signal analytique pur correspondant à chacun d'entre eux. C'est la chimiométrie et l'exemple le plus connu consiste à créer des réseaux de reconnaissance olfactive (les "nez électroniques") (2). C'est là une possibilité importante car elle résoud le problème absolument fondamental que pose la sélectivité pour les capteurs chimiques, problème qui a toujours freiné leur développement et que ne résoud évidemment pas la simple diminution de taille. Contrairement à la biologie et la biochimie et à leurs réactions naturellement sélectives qui mettent respectivement en jeu les couples enzyme/substrat et anticorps/antigène, la chimie ne concerne que des molécules et des ions trop voisins les uns des autres pour être distingués, "reconnus", facilement. La conjugaison de réseau de microcapteurs imparfaits associés à des circuits de traitement du signal puissants est une réponse à ce problème qui prendra certainement du poids dans les années à venir.

\section{II - METHODES de MINIATURISATION}

La tendance actuelle est à la miniaturisation des capteurs, chimiques, électrochimiques et autres. Les procédés de miniaturisation emploient un ensemble de techniques, issues de la microélectronique, qui ont une forte imprégnation chimique puisqu'il s'agit de modeler, de mettre en forme des structures tridimensionnelles composées de matériaux métalliques, semiconducteurs ou diélectriques. Elles consistent à effectuer, successivement, des opérations de dépôt ou d'érosion de matériaux selon une séquence appropriée dans des microzones délimitées par les procédés de la photolithographie. Un bref rappel du principe des méthodes employées pour la fabrication des microcapteurs est fait ici.

\section{1 - La photolithographie (3-5)}

Tous les procédés de miniaturisation reposent sur la capacité que l'on a de pouvoir transférer, par projection lumineuse, l'image d'un masque opaque, qui délimite les formes à obtenir, sur les substrats à traiter (généralement, ici, des tranches de silicium ou de quartz). Le résultat est obtenu grâce à des résines photosensibles, les photorésists. Les parties insolées par des rayons U.V. voient leur cohésion chimique et leur insolubilité renforcées par réticulation ou affaiblies par formation de groupements hydrophiles, selon qu'elles sont "négatives" ou "positives". Ceci conduit à une dissolution sélective des motifs les plus solubles. On peut ainsi "ouvrir" des fenêtres de géométrie définie par la forme du masque et accéder, de façon sélective, au matériau sous-jacent. C'est la base de la technologie "planar" de fabrication des circuits intégrés en silicium. Dans l'exemple de la figure 1, on dissoudra par exemple la silice rendue accessible par de l'acide fluorhydrique afin d'avoir accès au silicium que l'on soumettra alors au traitement suivant (attaque corrosive, dépôts divers). La taille usuelle des microcapteurs fait que la résolution inférieure au micromètre, de ce procédé suffit généralement et qu'il n'est pas besoin, sauf pour la technique LIGA que nous verrons plus loin, d'utiliser les procédés plus résolutifs, utilisant des rayonnement $X$ ou des faisceaux d'électrons, qui sont utilisés en microélectronique à très forte intégration. De ce fait les motifs n'excèdent par quelques micromètres en hauteur. 


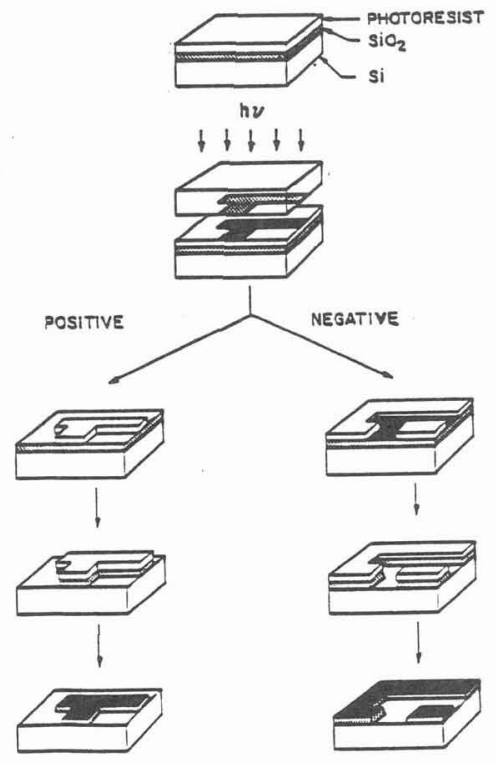

Fỉg. 1 Procédé photolithographique de reproduction d'un motif
D'un point de vue pratique, la résine est étalée sur l'échantillon que l'on dépose sur un plateau horizontal (la tournette) tournant à une vitesse déterminée dans une atmosphère à température contrôlée. De la sorte, la viscosité de la résine est reproductible ce qui conduit à des épaisseurs contrôlées. C'est le spin-coating.

Les motifs étant de dimension comparable à celle des poussières, cette opération comme toutes les autres opérations de microfabrication, se font généralement dans des "salles blanches" dépoussiérées.

\section{2 - Les dépôts de matériaux en couches minces (de l'ordre de quelques micromètres ou moins)}

La méthode de loin la plus utilisée est la méthode CVD (Chemical Vapour Deposition). Dans un réacteur à parois étanches et généralement sous basse pression (LPCVD), les substrats à traiter sont mis en présence de gaz qui réagissent à leur contact en conduisant au matériau souhaité. Afin de pouvoir opérer à température plus basse, un apport d'énergie peut être fait par emploi d'un plasma (PECVD) ou de photons (EECVD). Le polysilicium, largement utilisé dans les microstructures, est ainsi obtenu par réaction du $\mathrm{SiH}_{4}$ à $400^{\circ} \mathrm{C}$, tandis que la silice l'est, à $450^{\circ} \mathrm{C}$, par l'action d'un mélange de $\mathrm{SiH}_{4}$ et de $\mathrm{O}_{2}$. Comme ce procédé est largement utilisée pour le dépôt de diélectriques, il faut noter ici une méthode qui constitue le point de départ du traitement de tout substrat en silicium, celle de son oxydation tnermique, en atmosphère sèche ou humide d'oxygène, qui permet la croissance extrêmement régulière de silice thermique.

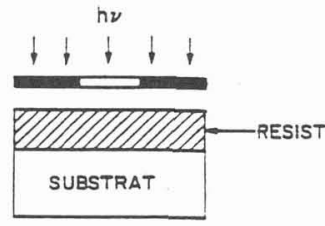

(a)

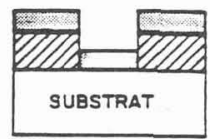

(c)

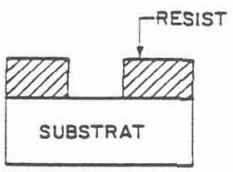

(b)

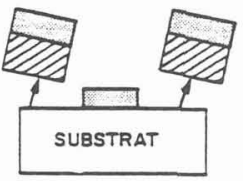

(d)

Fig. 2 Procédé lift-off

Une autre méthode de croissance de couches d'oxydes, très simple est actuellement en faveur, c'est la méthode du dip-coating qui repose sur une approche sol-gel. Des alcoxydes métalliques appropriés sont dissous dans un milieu alcoolique et utilisés comme précurseurs. L'échantillon à recouvrir est plongé dans la solution puis lentement extraite pour obtenir, par hydrolyse-polycondensation à la vapeur d'eau ambiante, la couche souhaitée. Un traitement thermique est ensuite nécessaire pour convertir le gel en composés oxydés. Les épaisseurs atteintes sont de 100 à $300 \mathrm{~nm}$.

Le spin-coating a été déjà décrit pour l'étalement des photoresists. Il est aussi largement utilisé, entre autres, pour l'étalement de polymères dissous dans un solvant que l'on évapore ensuite. 
Pour le dépôt de métaux, la méthode la plus utilisée est l'évaporation thermique. Elle est conduite en enceinte maintenue à basse pression $\left(10^{-4}\right.$ à $10^{-7}$ torrs), l'évaporation étant obtenue par effet Joule (fil résistant de tungsène ou molybdène). La cible est disposée en regard de la source d'atomes métalliques. La figure 2 montre que le dépôt d'un métal sur des motifs de résine en relief se fait sur toute la surface. En décollant la couche de résine par immersion dans un solvant, celle-ci se brise à la jonction amincie des fenêtres. Ce procédé, largement utilisé, est le procédé "lift-off". D'autres méthodes que la méthode thermique sont utilisées pour la pulvérisation atomique, par exemple le bombardement ionique ( sputtering) ou électronique.

\section{3 - Les dépôts de matériaux en couches épaisses}

Sous sa forme usuelle, la sérigraphie est un procédé qui permet de transférer un motif opaque, dessiné sur une trame de fils entrecroisés, sur un substrat à l'aide d'une pâte que l'on force à travers les parties libres de celle-ci. Par son principe, la méthode ne nécessite pas de procédés photolithographiques mais la résolution latérale est bien moindre. Une cuisson ultérieure permet de solidifier la pâte qui a traversé les motifs. C'est un procédé très utilisé pour les dépôts de céramiques. Certaines pâtes (ou encres) actuelles sont photosensibles ce qui rapproche désormais ce procédé de la photolithographie. Des épaisseurs supérieures à $100 \mu \mathrm{m}$ peuvent ainsi être obtenues.

\section{4 - L'érosion des matériaux (etching) (3-5)}

Elle est réalisée soit de façon ionique en milieu gazeux (érosion par plasma), soit en milieu liquide (érosion chimique).

Dans la première de ces méthodes, les matériaux sont arrachés, dans les fenêtres photolithographiées, par bombardement d'ions. Les couches de résine résistent à cette attaque tandis que des puits profonds de plusieurs centaines de nanomètres peuvent être creusés avec un fini de surface médiocre. L'érosion peut avoir une nature essentiellement mécanique quand les ions projetés sur la surface par un champ électronique sont inertes, comme les ions argon. Elle peut avoir une nature chimique si des molécules plus réactives sont employées. L'érosion du silicium est ainsi obtenue par un plasma $\mathrm{SF}_{6}+\mathrm{Cl}_{2}$ et celui de la silice par un plasma $\mathrm{CF}_{4}+\mathrm{H}_{2}$.

Dans la seconde de ces méthodes, le milieu d'attaque liquide prend le nom d'etchant. L'attaque se fait par simple immersion dans ce milieu. C'est une corrosion oxydante qui, si l'attaque est rapide, conduit à une dissolution isotropique et qui, si elle est lente, conduit à une dissolution anisotropique durant laquelle les plans cristallins des monocristaux peuvent être distingués grâce à des vitesses d'attaque très différentes (6-7). C'est ainsi que pour le silicium monocristallin, l'etchant rapide usuel type est le CP-4A, mélande d'acide nitrique, d'acide fluohydrique et d'acide acétique, alors qu'une solution aqueuse à $44 \%$ de potasse à $85^{\circ} \mathrm{C}$ a une action fortement anisotrope. Dans ce dernier cas la vitesse d'érosion des plans $<111>$ est plusieurs dizaines à plusieurs centaines de fois inférieure à celle des plans $<110>$ et $<100>$. Quand le plan de la tranche de silicium à traiter est le plan $<100>$, en pratiquant des fenêtres dont les côtés sont parallèles au plan $\langle 110\rangle$, on obtient, par exemple, des ouvertures en forme de sillon d'inclinaison, par rapport à la verticale, de $35^{\circ} 3$. 


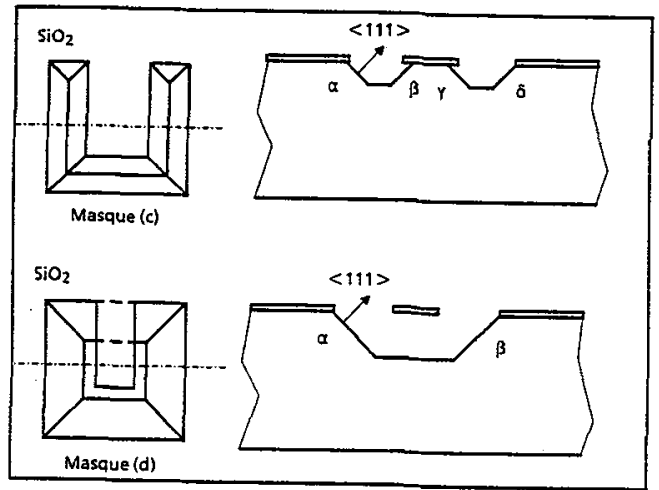

Fig. 3 Principe de réduction d'une poutre en silice en surplomb
En utilisant des masques de forme appropriée, en variant les plans d'attaques et la forme des fenêtres, il est possible, par cette méthode de dissolution anisotropique de construire des formes tridimensionnelles, limitées cependant en hauteur par l'épaisseur de quelques micromètres de la résine. La figure 3 , empruntée à la référence 7 , montre le principe de réalisation d'une poutre en surplomb en silice.

\section{5 - Méthode de fabrication de microobjets par la méthode des couches sacrifiées (7)}

En jouant sur l'empilement de couches successives, le plus souvent réalisées par CVD, en matériaux diélectriques $\left(\mathrm{SiO}_{2}, \mathrm{Si}_{3} \mathrm{~N}_{4}\right)$ ou métalliques ( $\mathrm{Al}$ ) ou encore en silicium polycristallin et l'usage de solvants sélectifs, il est possible de dissoudre certaines d'entre elles en libérant des pièces en matériau insoluble.
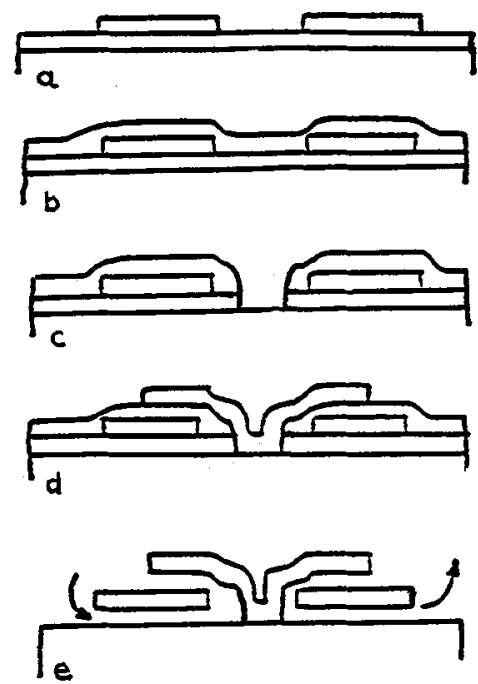

Fig. 4 Fabrication d'une pièce tournante. Après dépôt, sur une couche de silice thermique, de ce qui sera la partie tournante en polysilicium (a) et recouvrement par de la silice (b), l'axe du rotor en polysilicium est mis en place (c) puis déposé (d). La pièce tournante est ensuite dégagée par dissolution de la silice.
On fabrique, comme ci-dessus, des objets plats plutôt que réellement tridimensionnels. Les pièces dégagées sont généralement en polysilicium et les couches éliminées en silice. La figure 4 montre comment dégager une pièce tournante, de symétrie circulaire. C'est une véritable révolution technologique qu'a introduite cette méthode depuis quelques années. Elle s'est illustrée par la fabrication de micromoteurs électrostatiques de quelques centaines de micromètres de diamètre $(8)$. 
6 - Méthode et fabrication de microobjets par la méthode LIGA (Lithographie, Galvanoformung. Abdformung)

Les méthodes de fabrication précédemment évoquées présentent l'inconvénient de n'autoriser que des reliefs faibles. La technologie LIGA, d'origine allemande, pallie cet inconvénient : en utilisant non plus des rayonnements ultra-violets mais des rayons $X$ et des résines appropriées de type polyméthylméthacrylate. Des objets ayant une épaisseur de plusieurs centaines de micromètres peuvent ainsi être fabriqués. Des sources intenses de rayons $X$ sont nécessaires pour éviter des opérations trop longues et on est ainsi amené à utiliser des rayonnements synchrotrons. Le procédé est couteux.

La fabrication des objets passe par le remplissage des ouvertures pratiquées dans la résine par du métal $(\mathrm{Ni}, \mathrm{Cu})$ déposé électrolytiquement. Quand de nombreux objets doivent être fabriqués, on opère en injectant une résine dans cette matrice métallique et en utilisant cette forme, en négatif, comme un moule dans lequel on fait une nouvelle croissance électrolytique de métal.

A cet inventaire bref, il convient d'ajouter quelques techniques particulières qui permettent de faire des opérations de fabrication spéciales. Cest le cas, par exemple, de la fabrication de silicium poreux (9) par oxydation anodique du silicium en milieu acide fluorhydrique. L'érosion du matériau se fait alors sous forme d'un réseau de canaux, de quelques dizaines de nanomètres au plus. Par oxydation thermique, cette structure est stabilisée ce qui permet de l'utiliser comme poreux (10). On connait l'importance de ceux-ci en électrochimie. C'est aussi le cas de la soudure verre-silicium, ou silicium-silicium, qui permet de souder de façon électrostatique (et chimique) des plaques de matériaux une fois ceux-ci usinés (5).

\section{III - LES MICROCAPTEURS}

A l'aide de l'arsenal des méthodes de microfabrication dont on dispose, il devient possible de réaliser les microobjets souhaités.

L'analyse de la bibliographie très récente montre que deux voies sont actuellement suivies pour miniaturiser les capteurs. La première consiste en une simple réduction de taille de macrocapteurs éprouvés. La seconde repose sur la sensibilisation chimique, ou "fonctionnalisation", de dispositifs permettant de traduire des modifications subies par un élément de reconnaissance (la sonde ou encore le récepteur) lors de son contact avec des espèces à détecter, en un effet physique qui est toujours, en dernier ressort, un signal électrique. Ce dispositif de traduction s'appelle un transducteur.

Comme il est rare que les conditions d'utilisation des microcapteurs exigent un niveau de miniaturisation comparable à celui des microcircuits intégrés, un capteur ayant des dimensions millimétriques peut, en fait, être considéré comme un microcapteur. Les technologies de miniaturisation ne sont pas, ici, poussées à l'extrême. 


\section{1 - La miniaturisation des capteurs existants}

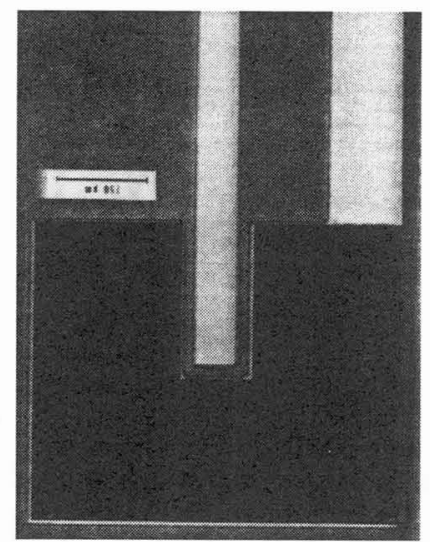

Fig. 5 Electrode planaire de CLARK (d'après réf. 12)

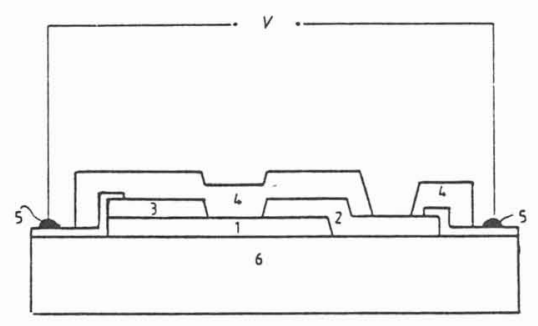

Fig. 6 Capteur zircone

1.Film CSZ - 2. Electrode de travail en platine poreux - 3. Référence $\mathrm{Pd} / \mathrm{PdO}_{\mathrm{x}}$ - 4. Film épais d'enamel - 5. Contacts électriques (d'après réf. 13)
Un premier exemple est fourni par l'électrode ampérométrique classique de CLARK (11) destinée au dosage de l'oxygène dissous par réduction cathodique de celui-ci. Une réalisation planaire de ce capteur est due au Centre Suisse d'Electronique et de Microtechnique (12). Sur un substrat de silicium oxydé sont déposées, en utilisant les techniques standards des circuits intégrés, deux électrodes en couche mince, une cathode d'argent centrale entourée par une anode $\mathrm{Ag} / \mathrm{AgCl}$. Un hydrogel est utilisé comme électrolyte, il est maintenu en place par une membrane de silicone qui assure la pénétration de l'oxygène (Fig. 5). emple est fourni par un microcapteur à oxygène gazeux à base de zircone (13). Dans ce capteur, la couche d'électrolyte solide (ici CSZ) est réalisé par sérigraphie (Fig. 6).

L'arrivée de l'oxygène à l'électrolyte CSZ, conducteur de $\mathrm{O}^{--}$, se fait à travers une électrode poreuse de platine ce qui permet à la cellule de fonctionner en régime diffusionnel. La référence est constituée par une électrode $\mathrm{Pd} / \mathrm{PdO}_{\mathrm{x}}$.

Un dernier exemple est l'électrode enzymatique au glucose (14). Elle comporte une électrode de référence $\mathrm{Ag} / \mathrm{AgCl}$, une cathode où se fait la réduction de l'eau oxygénée provenant de la réaction enzymatique et une contre-électrode. Une membrane perméable au glucose, en polyuréthane, maintient la membrane chargée d'enzyme glucose oxydase (Fig.7). 


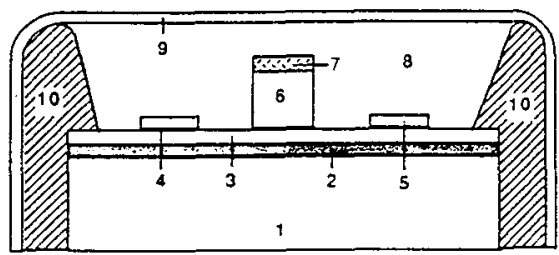

Fig. 7 Schéma d'un capteur au glucose

1. Substrat $\mathrm{Si}-2 . \mathrm{SiO}_{2}-3 . \mathrm{Al}_{2} \mathrm{O}_{3}-4$. Ti - 5 .

$\mathrm{Pt}$ - 6. Ag - 7. AgCl - 8. Membrane

enzymatique -9 . Membrane polyuréthane -10 .

Encapsulant epoxy.

\section{2 - La fonctionnalisation des microtransducteurs}

Les microtransducteurs les plus étudiés à l'heure actuelle sont les composants électroniques, que leur aptitude naturelle à l'intégration rend particulièrement intéressants, les dispositifs utilisant des matériaux piézoélectriques et les fibres optiques (1). Tous sont utilisables en milieu aqueux mais seuls les premiers sont de nature électrochimique. Bien que les composants passifs, résistances et capacités, soient largement utilisés, ce sont les composants actifs du type transistor à effet de champ qui prédominent. Le sigle anglais FET (Field Effect Transistors) est systématiquement utilisé pour les désigner et ils prennent le nom de capteurs ISFETs (Ion Sensitive FET) s'ils sont destinés à détecter les ions, d'ENFET's s'il s'agit de capteurs enzymatiques et d'IMMUNOFET's (15) (s'il s'agit de capteurs immunologiques). C'est ce type de capteurs qui sera pris comme exemple de travail pour illustrer les différents modes de fonctionnalisation.

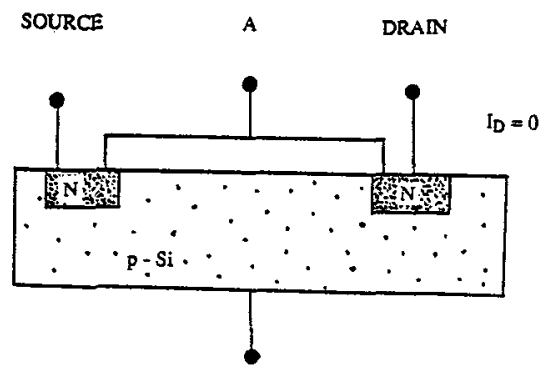

B
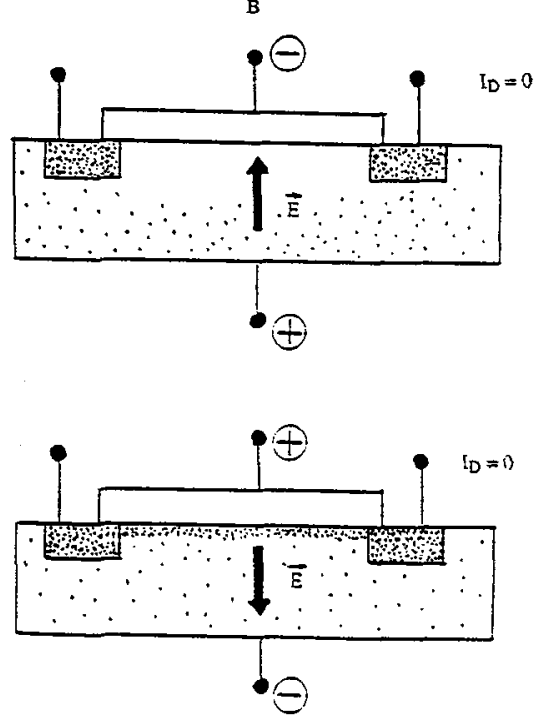

Un transistor à effet de champ en silicium est, en fait, une modification d'un composant MOSFET (Metal Oxide Semiconductor FET) tel que celui qui est représenté figure 8. Dans une tranche de silicium, généralement de type $\mathrm{p}$, deux enclaves fortement dopées $\mathrm{N}\left(\mathrm{N}^{+}\right)$servent respectivement de drain et de source pour les électrons qui circulent sous la grille en silice en créant un courant de drain id. Cet oxyde crée l'effet de champ dans le semiconducteur grâce à une polarisation adéquate appliquée entre sa métallisation superficielle et celle de la face arrière du composant. D'autres métallisations, celles du drain et de la source (contacts dits "ohmiques") assurent l'entrée et la sortie du courant ID qui deviendra le signal analytique. Dans la figure 8, la densité de points figure celle des électrons (zone $\mathrm{N}$ riche en électrons, zone $P$, pauvre en électrons). En l'absence d'une polarisation électrique entre $A$ et $B$, aucun courant ne passe entre drain et source puisque l'on a alors deux jonctions $p-n$ en opposition.En appliquant une polarisation $V_{G}$ négative modérée sur la grille, par rapport à la face arrière, le champ électrique repousse les électrons et la conduction sous celle-ci est toujours nulle.

Fig. 8 
Si on inverse cette polarisation, les électrons s'accumulent sous la grille (situation d'accumulation) dans une zone très mince, le canal, qui assure la conduction entre drain et source. L'apparition du courant de drain $\mathrm{I}_{\mathrm{D}}$ est obtenue pour une certaine valeur $\mathrm{V}_{\mathrm{T}}$ de la tension de grille, appelée tension seuil.

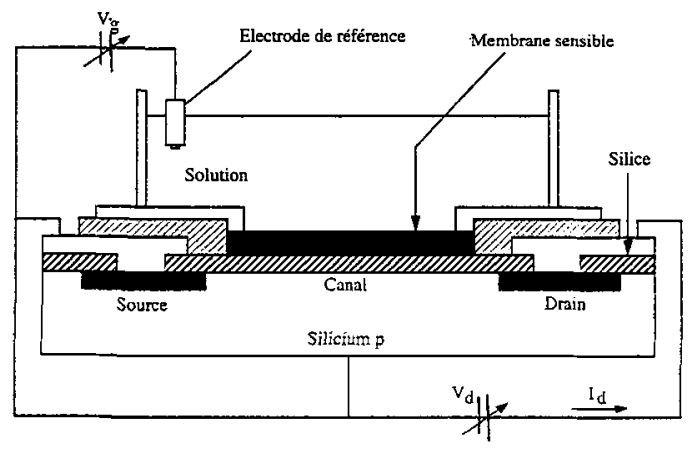

En supprimant la métallisation du diélectrique de grille, ces dispositfs deviennent capables de détecter des charges électriques pouvant exister à la surface de celui-ci, qu'il soit nu ou recouvert d'une membrane sensibilisatrice de reconnaissance. C'est ce qui est réalisé en milieu liquide où la continuité électrique entre la face externe modifiée du diélectrique et la base est assurée à l'aide d'une électrode de référence (Fig. 9).

Fig. 9 Schéma d'un ISFET

Dans la chaine du potentiel $V_{G}$ intervient la chute de potentiel, qui règne à la surface $d u$ diélectrique modifié. Si la fixation des espèces "reconnues", donc fixées à la surface réceptrice, entraine une variation de cette contribution, cela aboutit à une modification de la tension de seuil $\mathrm{V}_{\mathrm{T}}$ et à une translation de la caractéristique $V_{G} I_{D}$ des capteurs ainsi créés. De tels dispositifs peuvent donc fonctionner à $V_{G}$ ou $I_{D}$ constant.

Se pose dès lors le problème de la fonctionnalisation proprement dite. Comment peuton conférer des propriétés de reconnaissance à un diélectrique ? Diverses réponses ont été apportées à cette question qui sont du reste aussi utilisables pour les autres types de transducteurs évoqués plus haut (16-17). L'ouvrage de Janata sera consulté avec profit à cet égard.

\section{$\underline{\text { a }- \text { Mise à profit des propriétés chimiques de la surface des diélectriques }}$}

En milieu aqueux, les diélectriques usuels $\left(\mathrm{SiO}_{2}, \mathrm{Si}_{3} \mathrm{~N}_{4}\right.$, oxynitrures de silicium...) s'hydrolysent superficiellement en donnant naissance à des groupements silanols Si-OH (18). Cette hydrolyse s'observe aussi pour d'autres diélectriques tels que $\mathrm{Al}_{2} \mathrm{O}_{3}$ ou $\mathrm{Ta}_{2} \mathrm{O}_{5}$ (16). Les propriétés acido-basiques des groupements $\mathrm{X}-\mathrm{OH}$ ainsi formés conduisent à l'apparition d'une charge superficielle positive en $\mathrm{pH}$ acide (ou plus précisément à des $\mathrm{pH}$ inférieurs au point de charge nulle pzc) de ces surfaces par formation de groupements $\mathrm{X}-\mathrm{OH}_{2}{ }^{+}$et d'une charge négative aux $\mathrm{pH}$ plus élevés par formation de groupement X - $\mathrm{O}^{-}(19)$. Ces équilibres ioniques, entre la surface et les solutions d'immersion, conduisent à une réponse Nernstienne en potentiel aux protons, c'est-à-dire $59 \mathrm{mV}$ par unité pH. Des capteurs pH de ce type sont actuellement commercialisés, en particulier en France (20).

\section{$\underline{b}$-Dépôt de membranes réceptrices inorganiques}

De nombreuses membranes inorganiques ont été envisagées pour la détection des ions. Une membrane extrêmement prometteuse, par exemple, est constituée de NASICON ( $\mathrm{Na}_{3} \mathrm{Zr}_{2} \mathrm{Si}_{2} \mathrm{PO}_{12}$ ) (21) pour la détection des ions alcalins. Bien d'autres membranes, également insolubles, mettent ainsi en jeu un échange d'ions entre elles-mêmes et la solution, et de ce fait relèvent aussi théoriquement d'une réponse Nernstienne. C'est le cas, entre autre, de membranes en $\mathrm{LaF}_{3}$ (22) ou en verre aluminosilicate (23). Ces membranes sont généralement caractérisées par une bonne adhérence, ce qui 
est un paramètre important pour la longévité des capteurs, mais aussi malheureusement par un nombre relativement restreint de matériaux disponibles pour un tel usage.

\section{c-Dépôt de membranes réceptrices organiques}

Comme pour les macroélectrodes spécifiques, on recherche l'immobilisation de molécules réceptrices dans une membranes organique protectrice. L'exemple le plus répondu est celui des membranes PVC incluant des grosses molécules de valynomicine, antibiotique connu pour ses propriétés de reconnaissance des ions potassium (24). La méthode présente une plus grande diversité grâce au grand nombre de molécules ionophores disponibles (agents complexants, chelatants, ethers couronnes) mais souffre d'une adhérence généralement médiocre des membranes sur le substrat ainsi que d'une rétention souvent insuffisante des inophores dans la résine d'accueil. Se pose également le problème de la dissolution des plastifiants (16). Enfin, si la membrane est suffisamment épaisse, on peut observer une diminution de la conductivité sous l'isolant de grille ainsi qu'une augmentation des temps de réponse.

\section{d-Implantation d'ions}

Quelques résultats ont été publiés sur l'emploi de l'implantation ionique pour réaliser artificiellement de conducteurs ioniques. On peut citer, par exemple, les travaux de PHAM et al (25) sur l'implantation d'ions monovalents dans la silice. La méthode souffre d'un nombre très restreints de possibilités offertes et étudiées.

\section{e-Greffage chimique de molécules de reconnaissance}

Très récemment a été proposée une méthode de fonctionnalisation des substrats isolants, et en particulier de la silice, par greffage sur leur surface de molécules silanées à longue chaine (15-17) munies d'un groupement terminal de reconnaissance. Ceci est réalisé par réaction chimique de ces molécules avec les groupements silanols préalablement créés à la surface du diélectrique par un traitement sulfochromique.

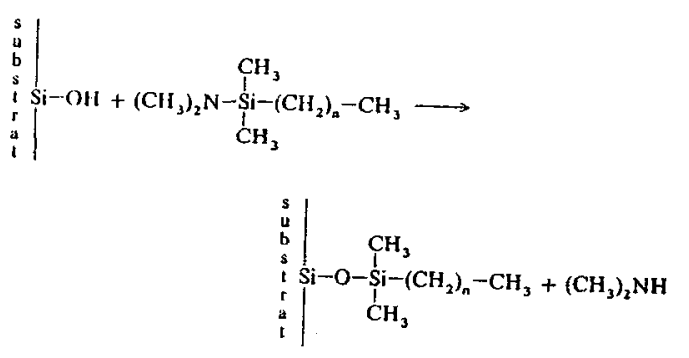

Dans l'exemple réactionnel de la figure 10 , le groupement méthyl en bout de chaîne devra être remplacé par le groupement de reconnaissance. Les chaines grasses $\left(C_{18}\right.$, par exemple) se réorganisent pour former un tapis hydrocarboné terminé par une monocouche des groupements fonctionnels choisis. Par ailleurs, la formation des liaisons covalentes Si-O-Si assure à la fois une parfaite continuité du substrat (si celui-ci est à base de silicium) et une solidité chimique remarquable.

Fig. 10

Très peu de travaux ont été jusqu'ici publiés sur cette méthode qui peut également servir à la formation de sous-couches pour la fixation des grosses molécules de reconnaissance biochimiques et biologiques (15). 


\section{IV - CONCLUSION}

L'ensemble des méthodes de fonctionnalisation des microtransducteurs permet d'envisager la réalisation de gammes de microcapteurs, malheureusement le plus souvent peu sélectifs, mais doués des qualités que l'on est en droit d'attendre des objets fabriqués par les méthodes collectives. On peut donc actuellement espérer que ces microcapteurs apportent dans un avenir proche une réponse aux innombrables problèmes analytiques qui se posent actuellement aux chimistes. Peutêtre en ayant recours à des réseaux de capteurs et au traitement mathématique de leurs informations.

\section{BIBLIOGRAPHIE}

1. Principles of Chemical Sensors. J. JANATA. Plenum Press. 1989

2. Sensors and Sensory Systems for an Electronic Nose. J. W. GARDNER et P.N. BARTLER Ed. Nato Asi Series, Serie E vol 212, 1992

3. The Chemistry of Semiconductor Industry. S.J. MOSS et A. LEDWITH Ed. Chapman and Hall, NewYork, 1987

4. Semiconductor Devices. Physics and Technology. S. M. SZE, John WILEY and Sons, New-York, 1985

5. W. H. KO et J.T. SUMITO dans "Sensors, a Comprehensive Survey". W. GÖPEL, J. HESSE et J.N. ZEMEL Ed., vol. 1 ; Fundamentals and General Aspects, VCH 1989 pp. 107-168.

6. K.E. PETERSEN, Proc. IEEE, 70 (1982) 420

7. Recherches en Microtechnique, Réalités et Perspectives. Collection du livre vert. 1992

8. L.S. FAN, Y.C. TAI et R.S. MULLER, Sensors and Actuators, 20 (1989) 41

9. I. RONGA, A. BSIESY, F. GASPARD, R. HERINO, M. LIGEON et A. MULLER, J. Electrochem. Soc. 138 (1991) 1403

10. R.L. SMITH et D.C. SCOTT, IEEE Trans. biomedical Engng., BME - 33 (1988) 83

11. L.C. CLARK, Trans. Am. Soc. Artificial Internal Organs (1956) 41

12. Scientific Report of CSEM, Maladière 71, CH 2000, Neuchâtel

13. G. VELASCO, J. Ph. SCHNELL et M. CROSET, Sensors and Actuators, 2 (1982) 371

14. M. KOUDELKA, S. GRENET et N.F. DE ROOIJ, Sensors and Actuators 18 (1989) 157

15. P. CLECHET, N. JAFFREZIC-RENAULT et C. MARTELET, Science Technique Technologies, 20 (1992) 40

16. P. CLECHET, Sensors and Actuators B4 (1991) 53

17. P. CLECHET, N. JAFFREZIC-RENAULT, Adv. Mat. 2 (1990) 293

18. Y. CROS, N. JAFFREZIC-RENAULT, J.M CHOVELON et J.J. FOMBON, J. Electrochem. Soc. 139 (1992) 507 
19. W. R. SIU et R.S.C. COBBOLD, IEEE Trans. Electron. Devices, ED 26 (1979) 1805

20. Catalogue "Bioblock Scientific 91" p.788

21. P. FABRY et E. SIEBERT, dans "Chemical Sensor Technology" vol.4 - KODANSHAN Ed. LTD (1992) p.111

22. N. MIURA, J. HISAMOTO, N. YAMAZOE, S. KUWATA et J. SALARDENNE, Sensors and Actuators 16 (1989) 301

23. M. ESACHI et T. MATSUO IEEE Trans. Biomedical Engng, BME 25 (1978) 184

24. S.M. MOSS, J. JANATA et C.C. JOHNSON, Anal. Chem. 47 (1975) 2238

25. M.T. PHAM, W. HOFFMAN et J. MÜLLER, Sensors and Actuators B8 (1992) 227 